\title{
ON THE PATHOLOGY
}

or

\section{TETANUS AND HYDROPHOBIA.}

\author{
BY \\ JOSEPH COATS, M.D., \\ IECTURER ON PATHOLOGY AND PATHOLOGIST \\ TO THE \\ WHSTERN INFIRMARY, GLASGOW.
}

Communicated by Dr. W. T. GAIRDNER.

- Received December 4th-Read December 11th, 1877.

Is the course of studying the histology of certain of the organs in cases of tetanus and hydrophobia, it has occurred to me that as these two diseases present many points of analogy, as well as many points of difference, they might be profitably studied together with a view to the elucidation of their pathology. It will not be denied that they present many points of analogy if not of similarity. They are both of them commonly related to some injury to the surface of the body. They both present, as their most characteristic features, certain nervous symptoms, of which the more prominent common characters are, a remarkable exaltation of reflex irritability, and the existence of groups of symptoms which may be referred to the medulla oblongata. There is, further, in all cases of hydrophobia, and in many cases of tetanus, a rise in temperature, which sometimes reaches a quite unusual height. 
I shall, in the first place, describe the pathological conditions which I have found in the organs examined, and then proceed to discuss the bearing of the facts obtained on the pathology of these diseases.

As to the material at my command, it is, in the case of tetanus, certain parts of the nervous system in five human cases of traumatic tetanus, and one case in the horse. In the case of hydrophobia I have examined the nervous system in two human cases and in one dog, and some of the internal organs in two dogs, the nervous system not being preserved in one of them. The structures were hardened in alcohol and a solution of chromic acid, and thin sections made with the razor. Sections were invariably examined in glycerine, but duplicates were usually coloured with carmine and mounted in dammar or Canada balsam, according to Lockhart Clarke's method. The two sets of preparations could therefore be used for comparison and correction.

\section{The Nervous System in Tetanus.}

The changes in the nervous system in this disease have been studied by several observers in this country and on the Continent, but we owe our knowledge of the condition of the spinal cord chiefly to Lockhart Clarke, Dickinson, and Clifford Allbutt. ${ }^{1}$

The spinal cord.-As to the condition of the spinal cord, I am in substantial agreement with the authors just mentioned. I find in my cases that every region of the cord presents, more or less, the following lesions:

1. There is great overfilling and distension of the bloodvessels, not uniformly, but at intervals. As the larger or medium sized nutritive vessels are situated mostly in the central parts of the cord and in the fissures, it is here, chiefly, that this condition is visible.

2. The most marked lesion is a granular condition around the vessels. This exists more or less in the neighbourhood

' 'Med.-Chir. Trans.,' xlviii and li, and 'Puth. Trans.' xxii. 
of nearly all the larger and moderately-sized vessels; and, as these are situated mostly in the central parts of the grey substance, and in the fissures, it is chiefly met with in these localities, although not confined to them. The characters of this lesion are best seen in specimens which have been mounted in glycerine. In these it is seen that the neighbourhood of the vessel is occupied by a mass of pretty coarse granules. This condition is shown in fig. 1 , and it will be seen that in addition to the granules, there are a number of much larger bodies of various sizes and mostly roundish in shape. These are bounded by a well-marked double contour, and are probably drops of myeline, as I have only met with them where the vessel was related to the white substance of the cord, chiefly in connection with the vessels of the fissures. It is a matter of dispute whether this granular material is an exudation or the result of disintegration of the nervous tissue. The appearances presented, especially in glycerine preparations, and shown in Plate VIII, fig. 1, are certainly very suggestive of an exudation; it often looks as if there were a kind of pool in the neighbourhood of the vessel, as if the material had been fluid in the living body, and these little pools sometimes run off to some little distance from the vessels. This view is confirmed by the occurrence of the granular material in the neighbourhood of the vessels which sometimes run transversely in the fissures; here there is sometimes a considerable gap between the granular material which sticks by the vessel and the neighbouring white columns. No doubt there is disintegration of nervous tissue, and the round bodies already mentioned and figured are most likely the myeline from nerve fibres which have been destroyed.

3. There is an appearance which hardly deserves to be separated from the last as a distinct lesion. In many of the sections the blood-vessels and surrounding granular material have fallen out, so that it is quite common to meet with gaps. These are usually round, but sometimes their outline is like the segments of two circles which have met, and it is as if two neighbouring lesions had coalesced. The gaps, voL. LXI. 
like the lesions they represent, are mostly in the neighbourhood of the central canal, that is to say, to either side of it in the grey commissure.

In one case I have met with a peculiar appearance in the white columns, to which, as it is isolated, I do not attach much importance. It is as if a cylindrical portion of white substance had become necrosed. In transverse sections there is a circular area, in which the nerve fibres and connective tissue do not take on the carmine, and this area is so loosely connected with the neighbouring white substance that in most sections it has dropped out. Of the many sections made, it only occurred in one set, but in these it was found continuous for some distance in the length of the cord.

These lesions were present in the horse as well as in man, although in the horse they were not so pronounced as in man. In the horse, only the medulla oblongata and upper cervical cord were obtained, but these parts were essentially in the same condition as those in the human cases. In the horse the central canal was filled with granular material, and apparently much distended. In the human cases the epithelium of the central canal seemed often unusually abundant; but as $I$ have found this so often under a variety of circumstances, I attach no special importance to it. The most marked example of so-called proliferation of the epithelium of the central canal which I possess is in a cord, which was hardened in order to give sections of the normal cord.

Medulla oblongata.-Lockhart Clarke did not find any lesion in the medulla oblongata in his original case, and Dickinson did not examine it microscopically. In all $\mathrm{my}$ cases the medulla oblongata was examined, and I have found it no less affected than the spinal cord. The three lesions described above were all present, though they may have varied in degree in different parts. In addition to these three lesions there were in this part pretty frequent small hæmorrhages, in which the blood corpuscles were frequently insinuated among the nerrous structures. These were 
not met with in the cord, although possibly they may have been present.

There is in the medulla oblongata a certain tendency to localisation of "the lesions, to which I am inclined to attach considerable importance, as we shall see further on. We saw that in the cord the larger and moderately-sized vessels run chiefly in the grey commissure and the fissures, and that the lesions exist chiefly there. In the medulla oblongata one of the most important nutrient vessels runs longitudinally in the posterior parts of the cord, to either side of and slightly behind the central canal or the deepest part of the floor of the fourth ventricle. In nearly every section this vessel is affected, and often there is hæmorrhage from it. It is well known that the nuclei of certain of the cerebral nerves are situated in this neighbourhood. The hypoglossal nucleus is the most prominent and easily recognised of these, and it is remarkable how often this nucleus and the bloodvessel with its lesion are visible in the same field of the microscope. It is not to be understood that the lesions were not present in other regions of the medulla. I have frequently met with gaps in the olivary body and elsewhere, but they are not nearly so common as in the region specially indicated.

The pons Varolii was only examined in one case, and it was found that though the above lesions were present, they were much less abundant than in the cord and medulla oblongata. The most aggravated hæmorrhage which I met with was in the pons, and there were evidences of irritation produced by the blood; that is to say, around the hæmorrhage and partly in it, there were large numbers of round cells, which are much more deeply stained with carmine than the red corpuscles or nervous tissue.

Corpora quadrigemina.-These ganglia were only examined in one case, but the characteristic lesions were very abundantly present, at least in one region. The appearances presented are well seen in a transverse section under a very low power. In such a section, made through the testes, there are seen to be several large gaps on either side of 
and behind, or above, the aqueduct. Here again it will be seen that the lesion follows the longitudinal vessels.

The corpus striatum was only examined in one case. There were a few gaps, but they were very infrequent, and the ganglion seemed to be involved in a very minor degree.

Convolutions.-These were examined in the last two cases which came under observation, and the parts chosen were the motor regions, as determined by Ferrier and Hitzig. In one of the cases the granular condition around the vessels was unmistakeably present but to a very slight extent. This was not found, however, in the other case. In both, there was a lesion which was not observed in the other regions of the nervous system, but which to a certain extent resembles the principal lesion found there. It consists of the exudation of a homogeneous yellow material outside many of the smaller blood-vessels (see Plate VIII, figs. 2, 3, 4, and 5). This condition was by no means homogeneously distributed, there being some parts of the convolutions where it did not seem to be present at all, and others in which it appeared to be present in connection with almost all the smaller vessels. It was only found in the grey matter of the convolutions, and in connection with vessels which were little if at all above capillary size. The exudation occurred in the form of little oval drops, and these retained their yellow colour in sections stained with carmine and mounted in the usual way in Canada balsam. I am aware that great care is necessary in interpreting appearances related to the vessels in the cortex of the brain, and my observations, so far as they have gone, confirm those of Obersteiner, ${ }^{1}$ who has shown that in what are regarded as normal brains collections of pigment exist immediately outside the vessels. Even in the brains under review it is very easy to find numerous examples of this aggregation of pigment around the vessels. But the lesion I have described and figured presents several points of difference from these. It occurs mostly in connection with the smaller vessels, while the ordinary pigmentary aggregations are commonly met with

1 Stricker's ' Jahrbücher', 1877, p. 231. 
on the surface of vessels of larger size than these. Then the ordinary pigment is granular, or contains granules, and lies in an elongated form along the outside of the vessel not apparently compressing it. The other lesion consists of a homogeneous material, having an appearance strongly suggestive of its being formed of a drop of some fluid; it has an oval form, and in nearly every case it compresses the vessel more or less, in many cases seriously compromising its lumen. It was this which first attracted my attention, and it is exceedingly striking to find, within a limited area, quite a number of vessels occluded more or less completely, in the way exhibited in figs. 2, 3, 4, and 5 .

Nerve.-The nerve proceeding from the injured part was only examined in one case, but no lesion was detected. There was no interstitial neuritis, such as some observers have found.

\section{The conditions met with in hydrophobia.}

As already mentioned I have obtained certain parts from two cases in man and two in the dog. The cases in man have been so fully reported in their clinical aspects by Drs. Dunlop and Patterson in the 'Lancet' of January 27th and February 3rd, 1877, that I need not refer further to them here. The cases were typical ones of hydrophobia.

It was with some difficulty that I obtained possession of the body of a dog which was said to have been affected with rabies, and only after it had been buried for a week. The symptoms in this dog were very carefully observed by $\mathrm{Mr}$. Alexander Henderson, who has considerable experience of dog ailments, and he has kindly given me a note of the facts observed. The symptoms are so well described, and the case seems such a typical one of rabies, that I shall here reproduce the account of them in his own words.

" On Thursday, 23rd November, 1876, I was called to see a dog about four years of age, which had returned home after 
an absence of four days in a state of great emaciation. Before going off it had been in its ordinary condition, which was that of a healthy dog but not fat. The owner of the dog thought that during its wanderings it must have been ill used. I found the dog lying on a sofa, and on my entrance, instead of, as usual, coming to meet me, he continued to rest. Its master said he could not understand its conduct. On being spoken to by its master, it gave no sign of recognition, but, on the contrary, snapped at him, then, all at once, would run to him, jump on his knee, and then as suddenly return to its sofa. The animal was a poodle of an extremely friendly temper, indeed an extraordinarily even temper. After noticing it for a short time I sat down on a stool and gave the dog my hand to smell, and when he had seen that there was nothing to fear from it, I laid hold of and muzzled him. The dog was very thin, extremely so ; and after a careful examination, the only mark I found on him was a little wound about a quarter of an inch below the left eye. Water, soup, and biscuits had been offered, and were placed before him, but he would have none of them. The animal showed a great dislike to light, and seemed to wish to be left alone in the dark. The following morning, Friday, I was sent for, and on arriving $I$ found the beast very much excited; through the night he had torn to pieces the sofa cover, and 'mouthed' the furniture in the room. It was some time before I could touch him; he was quite changed, and though seemingly desirous. of being alone, betrayed a constant watchfulness quite different to a dog wishing rest and quiet. He seemed very feverish and ill. I considered it unsafe to allow him to remain in the house, so removed him to a wooden outhouse, where I arranged a warm bed for him in the centre of the floor near the window. The animal, however, preferred to lie on the bare stones in a dark corner to having a comfortable bed in the light. I did not see him again till next morning, Saturday, when I found him much worse. $\mathrm{He}$ had still refused meat and drink although showing no dread of water, and at intervals he would get furious, crying and biting at the woodwork in an 
unreasoning manner, until utterly worn out he would drop on his side. The floor was covered with small pieces of wood, which in his rage he had torn from the side of the house. There was an absence of foam at the mouth, such as is met with in epileptic fits in the dog; but in this case a small quantity of thick adhesive saliva clung to the corners of the mouth, and seemed to worry the dog. Whether from this cause or not the animal was frequertly fighting with his paws at the corners of his mouth, the action being like that of a dog with a bone stuck in its throat, I thought it unsafe to allow him to live, so on Saturday afternoon I shot him. I consider this a genuine case of rabies or hydrophobia in the dog."

Of this dog I preserved the brain and spinal cord, the salivary glands, liver, kidneys, and spleen, and hardened them in alcohol and chromic acid. The other dog was seen during life by Dr. Klein, and he states that it was without doubt a case of rabies. The parts examined in it were the salivary glands, liver, spleen, kidneys, intestines, larynx, and trachea. I shall now proceed to describe the appearances presented in these various cases, and it is to be understood that, unless mention is made to the contrary, the appearances described appertained to the organs of all the cases which were examined.

The nervous system was examined in the two human cases, and in dog No. 1, and in all three the principal lesions were identical.

The principal lesion concerned the blood-vessels primarily, although its effects were not absolutely limited to them. Figs. 6 and 7 show the essential features of this lesion, and it will be seen that around the vessels there is a collection of round cells or leucocytes. In some cases there are only a few lying in the perivascular space, but in others they form a thick mantle, in which the vessel is completely buried beneath layers twenty or thirty deep. This condition does not affect the vessels uniformly; there is no continuous clothing of the vessels with leucocytes, but there appear to be free intervals alternating with spaces where the condition 
described is present. On the other hand, the lesion appears in some cases to occupy a considerable length of the vessel, as it is found that when a vessel happens to be exposed longitudinally, it is often seen to be clothed for a considerable distance with layers of leucocytes. In the dog's nervous system the vessels have often been torn out for some length in making the section, as the tissue was very brittle, and in these sections considerable lengths of blood-vessel are sometimes seen, clothed in a mantle of cells.

This condition is very abundantly present in the spinal cord and medulla oblongata. I am not convinced that any one region of the cord is more affected than another, but perhaps the dorsal region is less involved than the cervical. As the larger and medium-sized vessels of the cord run mostly in its central parts, and as it is chiefly these which are affected, we find that the lesion is most obvious in the grey commissure to either side of the central canal, but it is not confined to these parts, and may even be seen in the white substance. In the medulla oblongata it is present to a very marked extent, and indeed the most extreme aggregations of leucocytes which I have met with have been mostly here. In fact, it looks as if few of the vessels in the medulla oblongata had escaped. There does not seem to me to be so much localisation of the lesion in the posterior parts of the medulla as I found in tetanus, although to a certain extent a similar distribution is manifest.

In the pons Varolii the lesion is also present, and sometimes to a very marked degree, but it does not seem to be so frequent or so aggravated as in the medulla oblongata. In the corpora quadrigemina it was unequivocally present, but distinctly less frequent than even in the pons. The corpus striatum was, unfortunately, not preserved.

The convolutions were examined in one of the human cases and in the dog. There was here a marked contrast with the cord and medulla in respect that the larger and medium-sized vessels were nearly all unaffected, while in the case of those of nearly capillary size it was very common to meet with collections of leucocytes around them, and in 
some cases the vessel seemed to be buried in them and compressed.

It is here right to state that Benedikt, in a paper in Virchow's 'Archiv.' vol. lxiv, has, to a certain extent, anticipated these observations. He describes in the brains of dogs which had been affected with rabies, and in one human case, granular disintegration, an exudation of a hyaloid substance, and a kind of miliary abscess in connection with the vessels. The two first of these conditions I have not observed, but the condition just described may be taken to represent Benedikt's miliary abscesses. It is remarkable that, so far as I can discover, Benedikt says nothing as to the appearances in the cord and medulla oblongata, though it is there that I have found the lesion most pronounced.

Besides this lesion, which is the most constant and most noticeable in the central nervous system, there is a condition which seems closely related to it. The superabundant leucocytes are not confined to the neighbourhood of the blood-vessels, but in many parts of the nervous system they were seen to be present in unusual numbers in the tissue generally. The convolutions of the dog especially looked as if infiltrated with round cells. In some parts of the cord and elsewhere the leucocytes were aggregated around the ganglion cells, and occasionally they appeared to occupy the peri-ganglionic space in considerable numbers, as seen in Plate IX, fig. 10. It must be added that this latter is by no means a constant, or, indeed, a very frequent appearance.

The only other prominent abnormal condition observed in the central nervous system was an occasional great excess of amyloid bodies, chiefly near the surface of the corpora quadrigemina. As this, however, is not an unusual condition in other cases, I do not know that much importance is to be attached to it.

It may be added that I have not found any obvious hæmorrhages or areas of granular disintegration. I do not mean to say that they do not occur, but I have seen no 
unequivocal evidences of them, such as $I$ met with in tetanus.

The salivary glands excited most interest next to the nervous system. They were not preserved in the human cases, but in the two dogs they were examined, and in both the same conditions were observed. The glands were everywhere infiltrated with multitudes of round cells (see fig. 11), but the infiltration was greater at some parts than others. They were insinuated in large numbers between the proper glandular structures, but they had accumulated especially in the spaces occupied by the ducts and vessels. In these spaces their numbers are frequently so great as to obscure the outline of the vessels, which appear, as it were, buried in them. It is difficult to make out what relation these cells bear to the vessels, chiefly because the veins and arteries, except the largest, are mostly empty, or, at least, their contents are difficult to make out. It is easy to distinguish the arteries from the thickness of their coats, but the veins are mostly collapsed and buried in the aggregations of round cells, so that the walls are, for the most part, invisible. After careful investigation, however, it can be said that in some places, at least, there is a special accumulation outside of, and along the veins, and even where this cannot so definitely be made out, it is indicated by the existence of elongated groups of cells, the great excess of round cells in the spaces occupied by the vessels being another fact pointing in the same direction.

It may be added that the pancreas was examined in one of the dogs, but no such lesion was found.

The kidneys were examined in both dogs. The most obvious condition here is a very marked but not uniform hyperæmia, existing both in the cortex and pyramids, but especially manifest in the veins which run between the cortex and pyramids. So great is the distension in some of these veins that they occasionally look like large sinuses. It is sometimes evident that in the distended veins the white blood-corpuscles are abnormally abundant. This is very manifest in the specimen from which Plate IX, fig. 12, is 
taken, in which layers of white blood-corpuscles lie along the wall, sometimes in several layers. There is no extensive extravasation of the white corpuscles, that is to say, there is no infiltration of the kidneys with leucocytes, such as we have found in the salivary glands. In addition to this hyperæmia, which, it should be remarked, is by no means general, but appears localised to certain parts, there are, in one of the cases, frequent hæmorrhages. These are in the form of small collections of blood, and have their seat chiefly, if not entirely, in the cortical substance. They are often pretty close together, but always of small size.

The liver was examined in both the dogs. In one there is a general interstitial hepatitis, evidenced by an increase of connective tissue in the course of the portal vessels-in fact, an early cirrhosis hepatis. This is, obviously, a chronic condition, and has nothing to do with the hydrophobia; it was absent in the other dog. Otherwise the livers were hyperæmic, but not very markedly so, and if there is any excess of leucocytes in the connective tissue it is very slight.

The mucous membrane of the larynx was examined in one of the dogs, and it was apparent that the mucous glands were infiltrated with leucocytes in a similar way to the salivary glands, but to a much less degree. The infiltration was nearly uniform, each acinus appearing as if surrounded by a layer of round cells.

The cicatrix of the dog-bite, and the nerve-branches for some distance up from it, were examined in one of the men. In the neighbourhood of the cicatrix there were evidences of irritation in the form of leucocytes which infiltrated the skin and subcutaneous tissue. The blood-vessels in the neighbourhood contained, in some cases, a granular material, which was evidently disintegrated blood-corpuscles. In other cases the vessels were partially occupied by transparent, globular bodies, which are often yellow in colour, and are, I believe, red blood-corpuscles altered in some way. This alteration of the red corpuscles is met with in inflamed structures, and may also be taken as an evidence of irrita. tion. 
Such being the facts observed, it remains now to consider briefly the bearing which they may have on the two diseases before us. In the first place, as to hydrophobia, we found in the central nervous system certain lesions which $I$ do not presume to regard as distinctive of that disease, but which may, I think, be fairly considered evidences of irritation. There were leucocytes aggregated around the vessels and infiltrated into the nervous tissue often in great numbers. But these evidences of irritation did not exist only in the nervous system. The salivary glands, in the cases in which these were investigated, presented appearances strictly analogous to those in the nervous system-leucocytes around the vessels and infiltrated between the glandular elements. The kidneys also presented changes, the vessels were much dilated, and, more important than this, there were immense aggregations of leucocytes inside some of the veins, and also hæmorrhages. The existence of signs of irritation in all these organs, and the direct connection of these signs with the blood-vessels, is exceedingly suggestive of the existence of some irritant in the blood which has acted on the vessels primarily.

In the cases of tetanus subjected to examination, there were in the nervous system appearances which, though by no means identical with those in hydrophobia, are still to my mind strongly suggestive of irritation, and of irritation acting out from the blood-vessels. There is an exudation around the vessels, or a granular disintegration, and this lesion certainly suggests some irritant within the vessels. It is also to be noted that, while the relation to the bloodvessels of the respective lesions in tetanus and hydrophobia is similar, the distribution of these lesions in different parts of the nervous system is also remarkably similar. In both the lesions occur around the larger and medium-sized vessels of the cord, around the vessels of the medulla oblongata in a high degree, and in the convolutions to a slight extent. In tetanus the other organs have not been examined, and it cannot be said whether there are evidences of irritation in them, 
In regard to the symptoms in these two diseases, few will deny that in hydrophobia they are related to some poison circulating in the blood, and attacking specially the central nervous system. In the case of tetanus this view, though supported by very high authorities, is not generally received. Looking, however, to the fact, that on the one hand the symptoms in both these diseases have a closely analogous anatomical distribution, and that on the other hand the lesions though different in kind are so similar in distribution, it seems to me very natural to suppose that in tetanus also there may be some poison circulating in the blood and causing disturbance. In this connection it may be said that the high temperatures observed in tetanus, sometimes reaching a startling elevation, are more suggestive of a general disease, these temperatures not bearing any constant relation to the exaggerated muscular contraction. 


\section{DESCRIPTION OF PLATES VIII and IX.}

Pathology of Tetanus and Hydrophobia (Dr. Jos. Coats).

\section{Puate VIII.}

Fic. 1. Tetanus. From a section of the medulla oblong. at its lower part. A collection of granular material is seen, in which are larger bodies, probably drops of myeline. (Hartn. vii, $3=250$ diams.)

Figs. 2-5. Tetanus. From the convolutions. Oval drops of a yellow material are visible outside the vessels, often compressing them. (Hartn. vii, $3=250$ diams.)

Fig. 6. Hydrophobia. From a section of the spinal cord. Aggregation of leucocytes around a vessel. (Hartn. vii, $3=250$.)

\section{Plate IX.}

Fig. 7. Hydrophobia. From a section of the med. obl. at the lower part of the fourth ventricle. A vessel as if clothed with leucocytes. (Hartn. vii, $3=250$.)

Fias. 8, 9. Hydrophobia. From the middle of the cervical region of the cord. Leucocytes around ganglion cells.

Fra. 10. Hydrophobia. From the middle of the cervical region. Leucocytes around ganglion cells. (Hartn. ix, $3=460$.)

Fra. 11. Rabies in dog. Section of parotid gland, showing leucocytes infiltrated between the glandular acini. (Hartn. vii, $3=250$.)

Fra. 12. Rabies in dog. Section of kidney. A vein highly distended, and with great aggregation of white corpuscles. (Hartn. iv, 3 , with draw-tube $=120$.) 
Plate. VIII.

Med Chir Trans. Vol. IXI

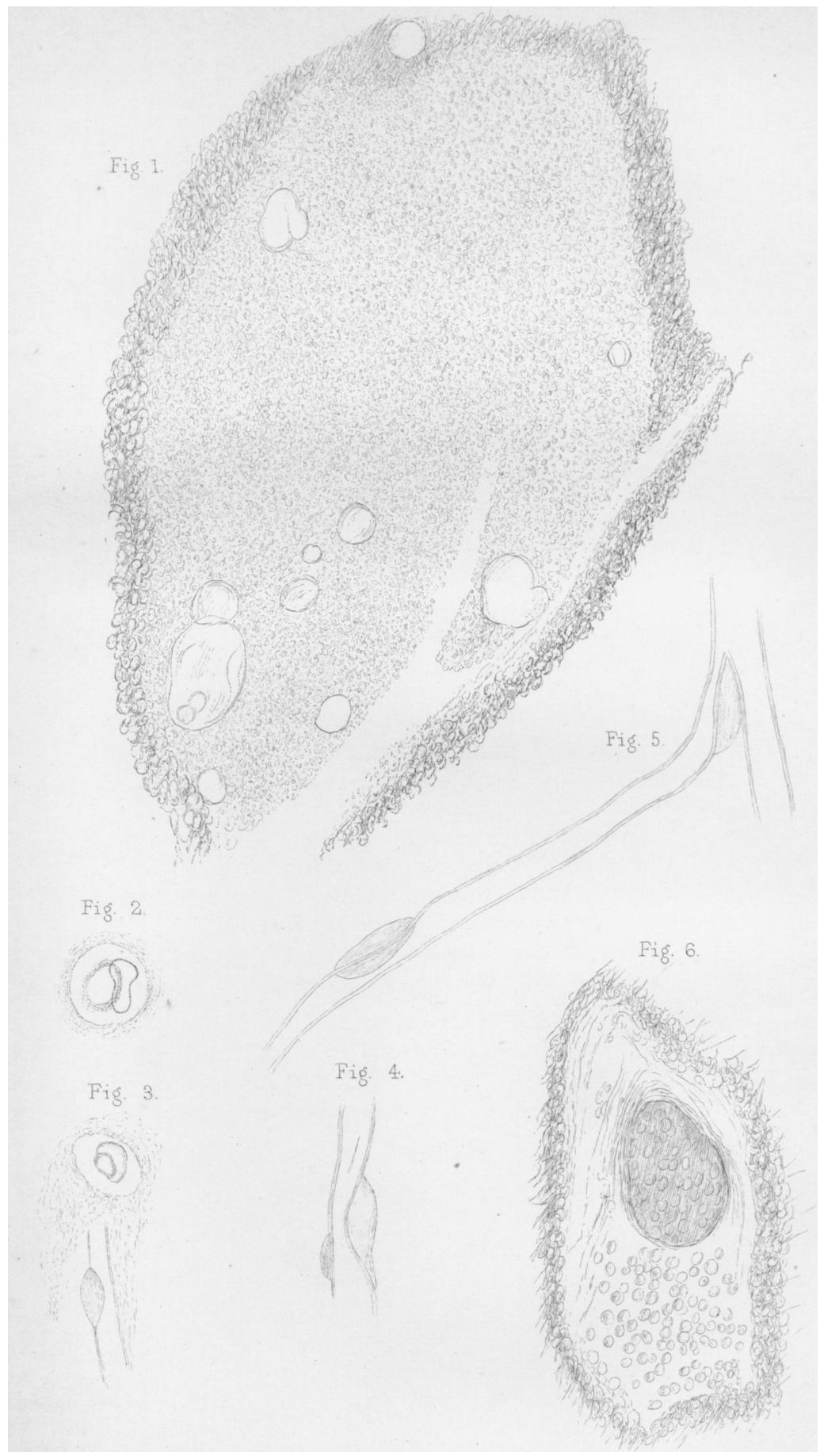




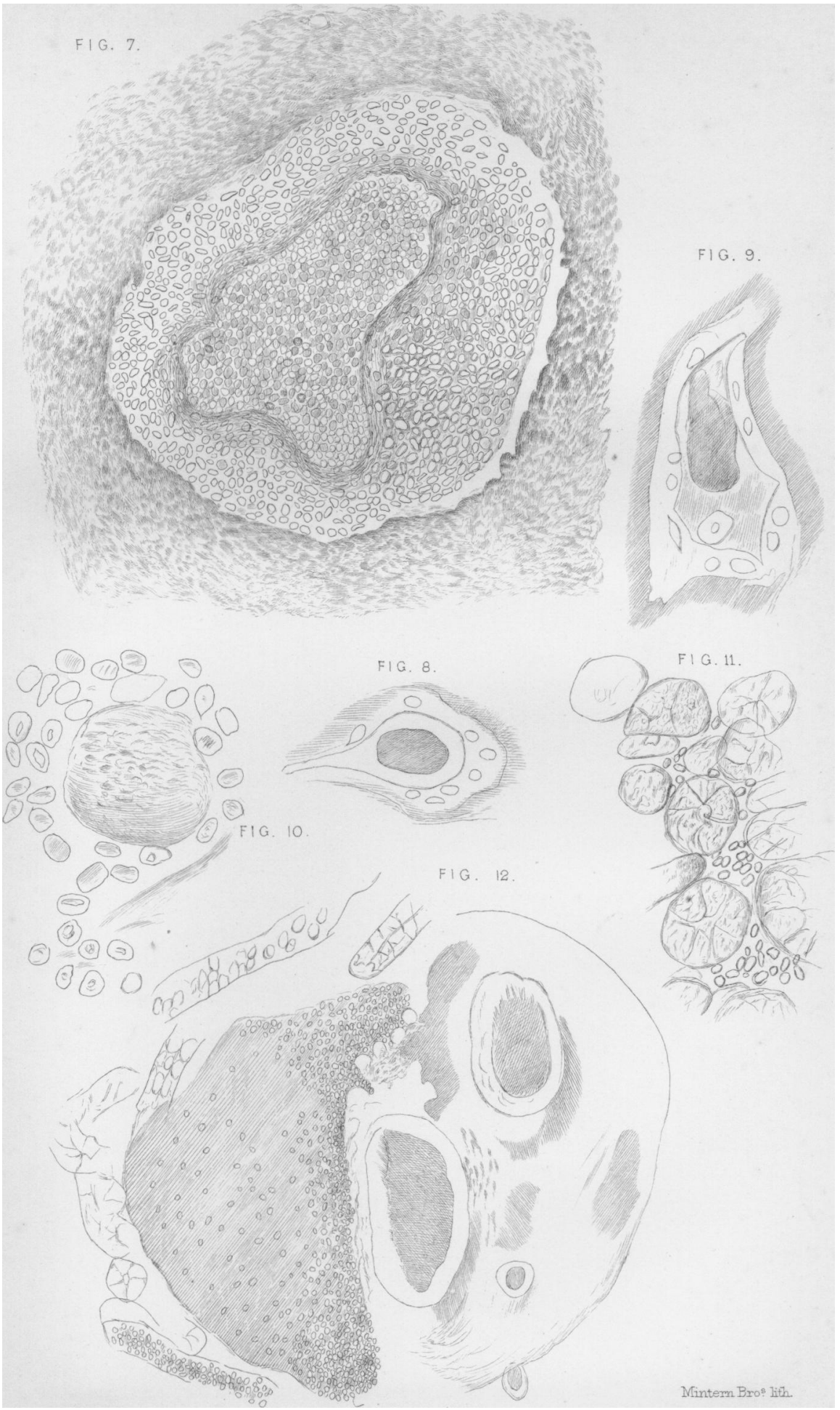

